\title{
Identification and characterization of the first cytokinin glycosyltransferase from rice
}

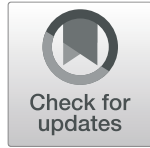

Pan $\mathrm{Li}^{1,2+}$, Kang Lei ${ }^{1 \dagger}$, Yanjie $\mathrm{Li}^{2}$, Xingrui He${ }^{1}$, Shuo Wang ${ }^{1}$, Renmin Liu', Lusha $\mathrm{Ji}^{1 *}$ and Bingkai Hou ${ }^{2^{*}}$

\begin{abstract}
Background: Cytokinins are one of the five major hormones families in plants and are important for their normal growth and environmental adaptability. In plants, cytokinins are mostly present as glycosides in plants, and their glycosylation modifications are catalyzed by family 1 glycosyltransferases. Current research on cytokinin glycosylation has focused on the biochemical identification of enzymes and the analysis of metabolites in Arabidopsis. There are few studies that examine how cytokinin glycosylation affects its synthesis and accumulation in plants. It is particularly important to understand these processes in food crops such as rice (Oryza sativa); however, to date, cytokinin glycosyltransferase genes in rice have not been reported.

Results: In this study, we identified eight rice genes that were functionally homologous to an Arabidopsis cytokinin glycosyltransferase gene. These genes were cloned and expressed in a prokaryotic system to obtain their purified proteins. Through enzymatic analysis and liquid chromatography-mass spectrometry, a single rice glycosyltransferase, Os6, was identified that glycosylated cytokinin in vitro. Os6 was overexpressed in Arabidopsis, and the extraction of cytokinin glycosides showed that Os6 is functionally active in planta.

Conclusions: The identification and characterization of the first cytokinin glycosyltransferase from rice is important for future studies on the cytokinin metabolic pathway in rice. An improved understanding of rice cytokinin glycosyltransferases may facilitate genetic improvements in rice quality.
\end{abstract}

Keywords: Glycosyltransferase, Cytokinins, Gene cloning, Prokaryotic expression

\section{Background}

Rice (Oryza sativa) is grown widely around the world and is the main food for more than $50 \%$ of the global population (Ramegowda et al. 2014). In recent years, with an increasing populations and environmental degradation, the land suitable for rice cultivation has diminished. To address these problems, the use of biotechnology to improve the stress resistance, yield and nutritional quality of rice is urgently required (Bansal et al. 2016). Cytokinins are important small molecule compounds, which are derivatives of adenine that occur naturally in plants (Plihalova et al. 2016). Cytokinins are one of the five major hormones found in plants, and are closely related to other plant hormones. Cytokinins

\footnotetext{
*Correspondence: jilu2060@163.com; bkhou@sdu.edu.cn

'Pan Li and Kang Lei contributed equally to this work

${ }^{1}$ College of Pharmacy, Liaocheng University, Liaocheng 252000, Shandong, China

${ }^{2}$ College of Life Science, Shandong University, Qingdao 250000, Shandong, China
}

affect plant growth and development (Zubo et al. 2017); including seed germination, chloroplast specialization, apical dominance, stress responses, cell differentiation and cell death. Therefore, the identification of the key enzymes of the cytokinin synthesis pathway in rice could be valuable for biotechnological applications. By functionally identifying these genes, their roles in the synthesis and metabolism of rice cytokinins, as well as in growth and environmental adaptation, could be further investigated. Better understanding these processes could lead to significant advances in the genetic improvement of rice yield and nutritional quality.

The first naturally occurring cytokinin identified in plants was zeatin, which was obtained from unripe corn endosperm (Frebortova et al. 2017). Later, as cytokinin research progressed, more and more types of cytokinins were identified. The naturally occurring cytokinins in plants can be divided into isoprenoid and aromatic forms on the basis of the type of cytokinin side chain. 
The molecular structures of the common active cytokinins were shown in Fig. 1 (Xu et al. 2017).

There are many cytokinin synthesis pathways in plants (Yamburenko et al. 2017). The pathways that have been identified to date can be divided into the transport RNA (Morrison et al. 2015) and de novo synthesis (Ciaglia et al. 2017). The transport RNA pathway produces too few cytokinins to meet the growth needs of plants. Therefore, the de novo synthesis pathway is the generally accepted main pathway for cytokinin synthesis. The de novo synthesis pathway also includes the adenosine monophosphate (AMP) pathway, the ATP/ADP pathway, and the alternative pathway. In the AMP pathway, dimethyl propylene diphosphate (DMAPP):AMP isoprenyltransferase catalyzes the transfer of an isopentyl group from dimethyl propylene diphosphate to the N6 position of AMP to produce a cytokinin with active pentyl adenosine-5-phosphate (iPMP) and isoamyladenosine groups (Ashihara et al. 2018). In the ATP/ADP pathway, the IPT4 enzyme obtained from Arabidopsis thaliana can preferentially utilize ATP and ADP as substrates in the presence of ATP, ADP and AMP. Isoprenoid adenosine-5-triphosphate and isoamyladenosine-5-diphosphate are possible reaction products of cytokinins, and can form zeatin through a further one-step hydroxylation reaction (Yu et al. 2015). The alternative pathway is an iPMP-independent pathway that transfers the hydroxylated side chain to the N6 site of adenine. This pathway was identified after it was discovered that the precursor of trans-zeatin nucleoside phosphate was not primarily derived from iPMP (Kaltenegger et al. 2018).

To date, studies of the cytokinin metabolic pathway have focused on Arabidopsis. The cytokinin receptors are three histidine kinases, AHK2, AHK3 and CYTOKININ RESPONSE 1 (also known as AHK4) (Lomin et al.

\section{A}<smiles>CC(C)=CCNc1ncnc2[nH]cnc12</smiles>

$N^{6}-\left(\Delta^{2}\right.$-isopentenyl)adenine

(iP)<smiles>C/C(=C\CNc1ncnc2[nH]cnc12)CO</smiles>

trans-zeatin

(tZ)<smiles>C/C(=C/CNc1ncnc2[nH]cnc12)CO</smiles>

cis-zeatin

(cZ)<smiles>CC(CO)CCNc1ncnc2[nH]cnc12</smiles>

dihydrozeatin

(DZ)

\section{B}<smiles>Oc1ccccc1CNc1ncnc2[nH]cnc12</smiles>

ortho-topolin

(oT)<smiles>COc1ccccc1CNc1ncnc2[nH]cnc12</smiles>

ortho-methoxytopolin (MeoT)<smiles>Oc1cccc(CNc2ncnc3[nH]cnc23)c1</smiles>

(mT)<smiles>COc1cccc(CNc2ncnc3[nH]cnc23)c1</smiles>

meta-methoxytopolin

(MemT)

Fig. 1 The molecular structure of common active cytokinins. a The molecular structure of Isoprenoid CKs. There are four forms of Isoprenoid CKs, $N^{6}$ - $\left(\Delta^{2}\right.$-isopentenyl) adenine (iP), trans-zeatin (tZ), cis-zeatin (cZ), dihydrozeatin (DZ). $\mathbf{b}$ The molecular structure of Aromatic CKs. There are five forms of Aromatic CKs, ortho-topolin(oT),meta-topolin(mT),benzyladenine(BA),ortho-methoxytopolin(MeoT), meta-methoxytopolin(MemT) 
2015). There are also more than 20 response regulators that are associated with cytokinins. These Arabidopsis response regulators can be broadly classified into four types: A-type, B-type, and C-type as well as Arabidopsis pseudoresponse regulators (Kurepa et al. 2014). In addition, the degradation of cytokinin mainly depends on the activity of cytokinin oxidative dehydrogenase, which irreversibly inhibits cytokinin degradation (Yeh et al. 2015; Xiao et al. 2016). Morris et al. (1999) found that cytokinins are extensively degraded in the anthers and pollen of cytokinin oxidative dehydrogenase transgenic corn, which eventually leads to male sterility during spore formation.

In nature, cytokinin compounds are found mostly as glycosides. Various cytokinin glycosides can be formed owing to differences in sugar types, attachment positions and aglycones. Further modification of these groups produces a wide variety of cytokinin compounds in nature and glycosylation is particularly important for this diversity (HIuska et al. 2016). Cytokinin glycosylation is mainly catalyzed by family 1 glycosyltransferases (GT1s) (Lao et al. 2014), which are conserved across different plant species. GT1s transfers the active sugar donor, usually a UDP-glycosyl group, to the hydroxyl group of the substrate to form a cytokinin glycoside product.

The cytokinin glycosides are still poorly understood. However, cytokinin glycosyltransferase genes have been studied in Arabidopsis. For example, Hou et al. (2004) used molecular and biochemical methods to demonstrate that the Arabidopsis glycosyltransferase UGT76C2 catalyzes cytokinin glycosylation in vitro. There are many substrates that can be catalyzed by glycosyltransferases, including free-form trans-zeatin, cis-zeatin, dihydrozeatin, isopentenyl adenine, 6-benzyl adenine and kinetin. After glycosylation, these mitogens form their corresponding N-glycosides. In 2011, Wang et al. (2011) conducted experiments in vivo using Arabidopsis thaliana as the model. They showed that UGT76C2 catalyzes cytokinin glycosylation in plants and participates in the regulation of cytokinins. The modifications made to cytokinins by glycosyltransferases may be considered as "fine tuning" the synthesis, metabolism and function of these compounds. Glycosylation may affect the transport of cytokinins in plants, the normal growth and development of plants, the specific distribution of cytokinins in tissues and cells, signal transduction processes associated with these molecules, and upstream regulatory factors. Therefore, studying cytokinin glycosylation is of great significance for understanding the metabolic regulation of cytokinin compounds and for clarifying their physiological effects.

Studies of cytokinin glycosyltransferase genes in rice have not previously been reported. Here, we selected eight candidate rice glycosyltransferase genes and found that one of these, Os6, encodes a protein that can glycosylate cytokinin in an in vitro enzymatic reaction, as determined by liquid chromatography-mass spectrometry (LC-MS). We transformed Arabidopsis with Os6 to obtain homozygous transgenic lines overexpressing Os6. We examined these plants for growth and developmental phenotypes, as well as analyzing their cytokinin synthesis, metabolic and signal transduction pathways. The candidate gene selected in this project, Os6, is the first rice gene reported to glycosylate cytokinin. This work lays a good theoretical and practical foundation that, with the ongoing study of Os6, will improve our understanding of cytokinin glycosyltransferase functions in rice. This should be beneficial for future genetic breeding approaches to improve rice quality.

\section{Results}

Screening and identification of rice cytokinin glycosyltransferase candidate genes

The complete rice genome of rice in now available through the rice genome database (http://rice.plantbiology.msu.edu). In 2008, Cao et al. constructed a phylogenetic tree of glycosyltransferases in rice using bioinformatics methods and identified 609 potential glycosyltransferase genes. Among them was GT1, which encodes the enzyme that transfers UDP-glucose. According to Cao's prediction, the family GT1 contains the largest number of genes (Cao et al. 2008). There are 8 and 41 genes with glycosyltransferase activity at the $\mathrm{N}$ - and $\mathrm{O}$ - positions, respectively, which may represent glycosylated cell classifiers, and the phylogenetic tree was constructed using MEGA software based on the similarity information of these sequences (Fig. 2). In our study, we selected eight candidate genes capable of glycosylating cytokinins, LOC_Os01g59100 (Os6), LOC_Os02g11130, LOC_Os07g30330, LOC_Os02g11700, LOC_Os07g30610, LOC_Os07g13800, LOC_Os11g25454 and LOC_Os07g13 810. LOC_Os01g59100 corresponded to the gene referred to as $0 s 6$ in this study.

\section{Spatiotemporal expression pattern of Os6 in WT rice and Os6 cloning}

To investigate Os6 expression in rice, we isolated various tissues and organs from WT rice plants at different developmental stages. RNA was extracted and Os6 expression was confirmed in various tissues and organs using qRT-PCR. Os6 was widely expressed to different degrees in various tissues and organs (Fig. 3), with a relatively high expression level in seedlings and a relatively low expression level in mature leaves. Then, the rice glycosyltransferase gene Os6 was successfully cloned from rice cDNA (Additional file 1: Figure S1a).

Construction of the prokaryotic expression vector and the Os6 protein's activity

After sequencing, the Os6 gene was cloned into a prokaryotic expression vector, PGEX (Additional file 1: Figure S1b). 


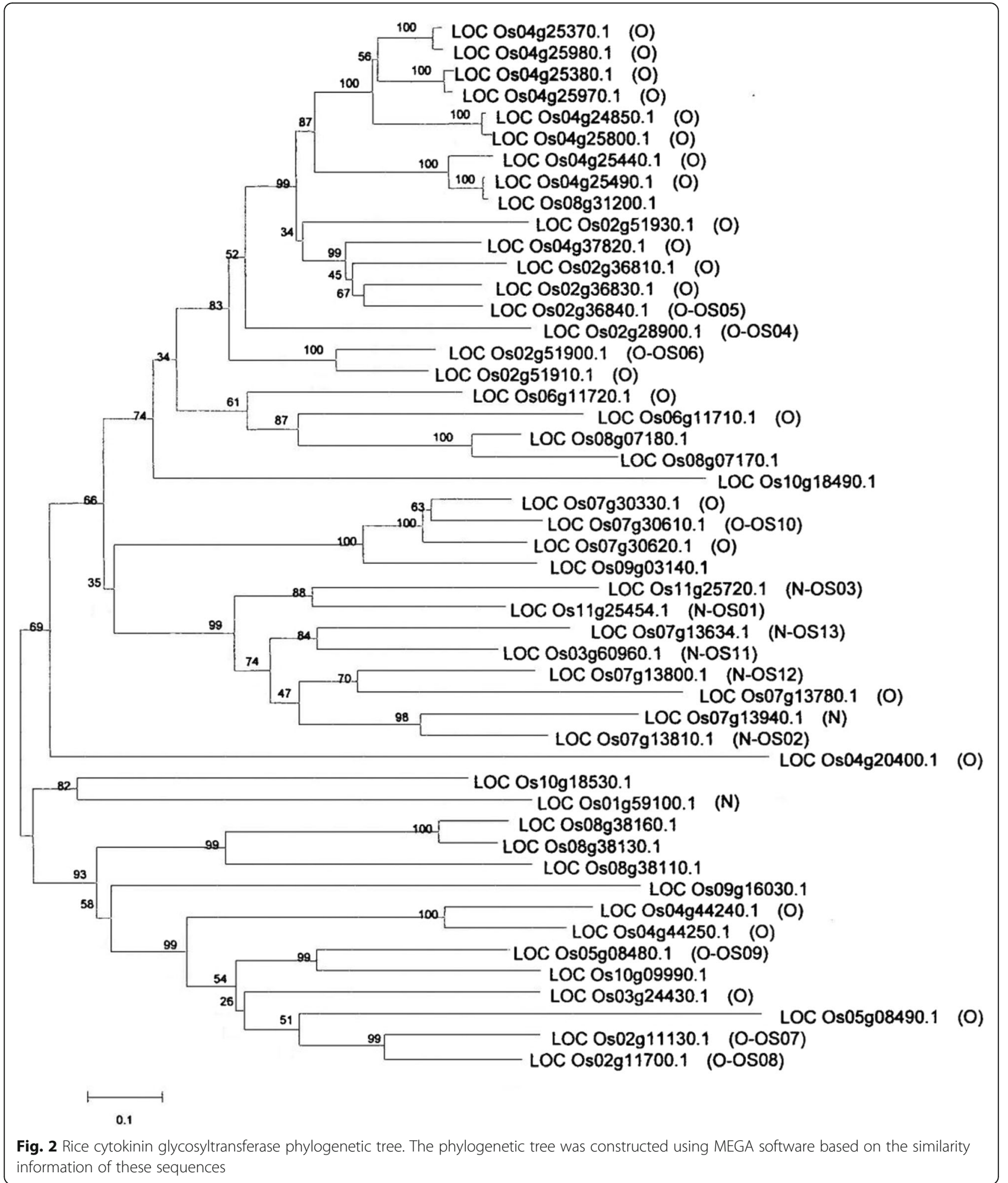

To investigate the Os6 protein's activity, we cloned the target gene into the PGEX vector, and then transformed it into the E. coli protein-expression strain XL1-Blue. Protein expression was induced and confirmed by sodium dodecyl sulfate-polyacrylamide gel electrophoresis (Fig. 4).
Rice glycosyltransferase Os6's enzymatic reaction and substrate identification

To investigate whether Os6 could glycosylate the plant hormone cytokinin, we tested this enzymatic reaction in vitro by mixing Os6 protein, cytokinin, buffer and 


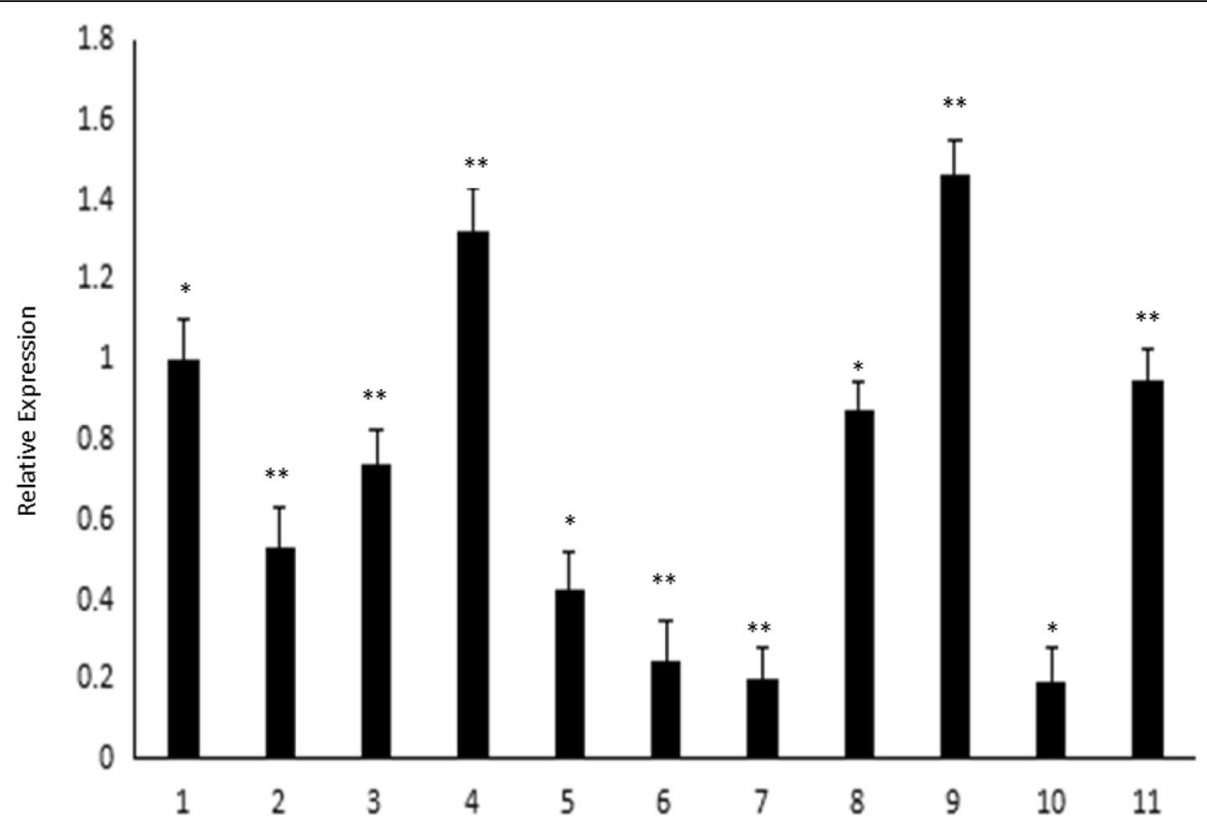

Fig. 3 Time and space expression pattern of Os6. In order to further understand the expression pattern of Os6, RNA was extracted from different tissues and organs of the wild-type rice plants, then Reverse transcription into CDNA, detection of Os6 expression pattern by Real-Time PCR. 1: Seedling Root; 2: Seedling Stem; 3: Seedling Leaves; 4: Seedling Sheath; 5: Mature Root; 6: Mature Stem; 7: Mature Leaves; 8: Mature Flower; 9: Mature Endosperm; 10: dried seeds; 11: wet seeds

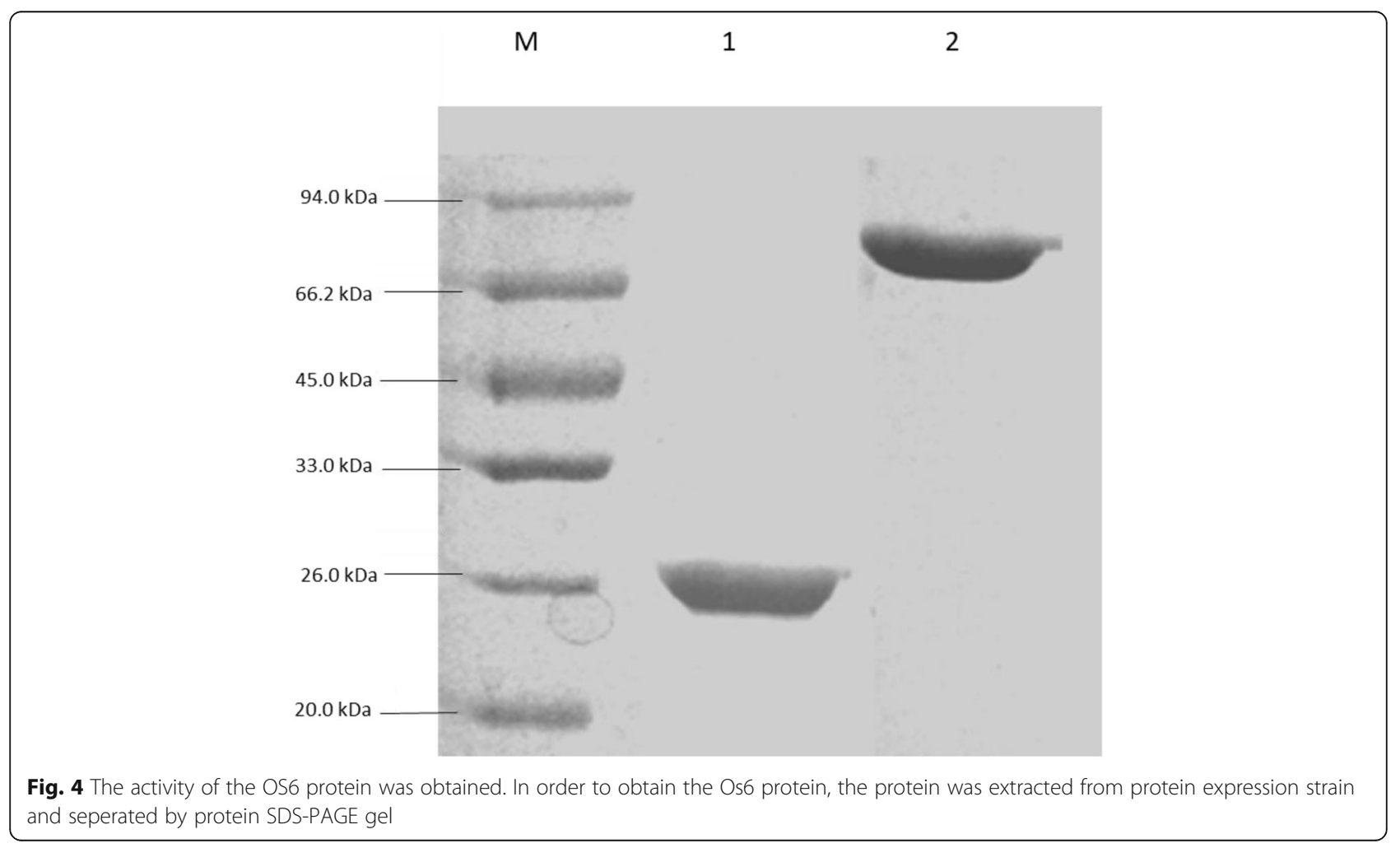


UDP-glucose. The products of the reaction mixture were examined using HPLC, and Os6 can transfer glucose to two cytokinins, trans-zeatin and 6-benzylaminopurine (Fig. 5a, b). The Arabidopsis glycosyltransferase gene UGT76C1 was used as a positive control. LC-MS was used to confirm the presence of glycosylated cytokinins produced by Os6 activity (Fig. 5c, d).

\section{Evaluation of the $\mathrm{K}_{\mathrm{m}}$ values of Os6}

Having found that Os6 could glycosylate cytokinins, we qualitatively analyzed the $\mathrm{K}_{\mathrm{m}}$ values of these cytokinin reactions using HPLC (Table 1). The $\mathrm{K}_{\mathrm{m}}$ values for Os6-mediated glycosylation of trans-zeatin and 6-benzylaminopurine were similar to those of UGT76C1.

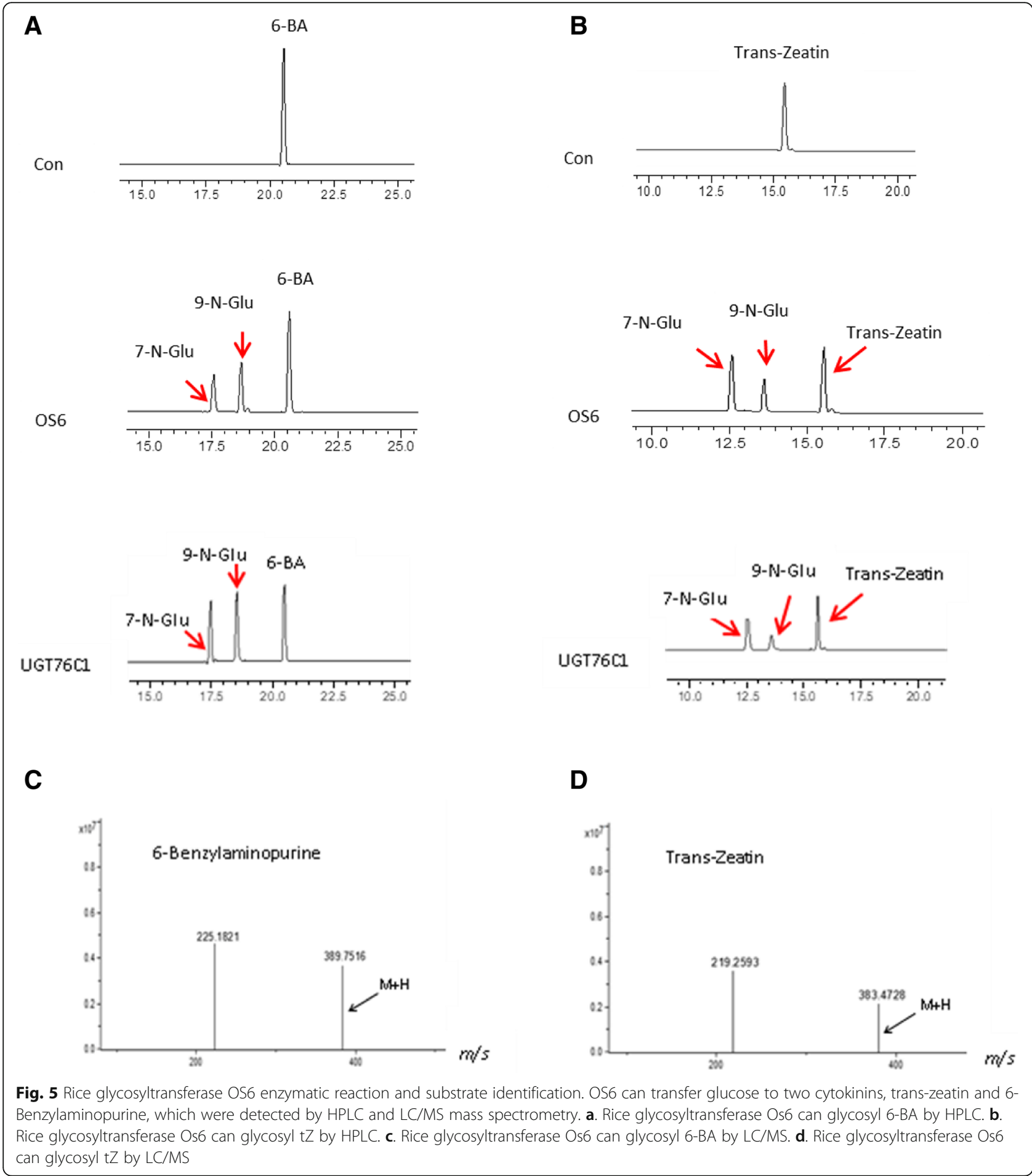


Table 1 The Km values of OS6, which glycosylate trans-zeatin and 6-Benzylaminopurine

\begin{tabular}{llllll}
\hline \multicolumn{5}{c}{ Specific activity (nkat/mg protein) } \\
\hline Trans-Zeatin & $76 \mathrm{C} 1$ & & Os6 & \\
& $7-\mathrm{N}$ & $9-\mathrm{N}$ & $7-\mathrm{N}$ & $9-\mathrm{N}$ \\
& & & & & \\
\end{tabular}

Os6 overexpression in Arabidopsis

To determine whether Os6 is functionally active in plants, we overexpressed Os6 in the model plant Arabidopsis. After three generations of screening, we obtained 25 transgenic homozygous lines. RNA was extracted and reverse transcribed into cDNA, and the expression of Os6 in each line was confirmed by qRT-PCR. Two highly overexpressing strains, OE4 and OE18, were selected for subsequent experiments (Fig. 6a).

\section{Cytokinin glycoside measurements in transgenic Arabidopsis}

The WT and transgenic Arabidopsis plants were grown under normal conditions for 2 weeks, after which the cytokinins and their glycosides were extracted using an organic solvent. Esculetin was used as an internal standard to quantitatively analyze each line by HPLC. The cytokinin glycoside content in the OE4 and OE18 overexpressing lines was greater than in the WT plants (Fig. 6b). Thus, the rice glycosyltransferase Os6 appears to glycosylate cytokinins in planta, and Os6 may act in the cytokinin pathway.

\section{Discussion}

In our research, first, we compared database information based on known conditions, and constructed a phylogenetic tree based on sequence similarity using MEGA software. The results are shown in Fig. 1. Owing to the lengths of the sequenced genes, the differences are large. The data analysis results showed obvious branches, but most rice glycosyltransferase genes were not associated with the already published cytokinin glycosyltransferase gene UGT76C1 and UGT76C2 in Arabidopsis (Smehilova et al. 2016) (Additional file 1: Figure S2). In our study, the eight studied rice cytokinin glycosyltransferase genes were randomly selected.

Because the other seven candidate genes are not glycosylated cytokinins, only Os6 can glycosylate cytokinins.
Then, we compared the gene sequence of Os6 with the genes of the Arabidopsis glycosyltransferase gene family members. However, there is no sequence homologous to Os6 in Arabidopsis. We also compared the sequence of Os6 with the sequences of UGT76C1 and UGT76C2, and found that the similarity was $\sim 40 \%$. After the analysis, we found that, in addition to the conserved 44 amino acids at the C-terminus of glycosyltransferase, there are other highly similar gene sequences, and we speculate that these sequences enable these genes to glycosylate cytokinins.

Here, we cloned a glycosyltransferase gene from rice and constructed a prokaryotic expression vector to purify the active enzyme protein. After adequate incubation, an in vitro enzymatic reaction was detected by HPLC. The rice glycosyltransferase Os6 was able to glycosylate cytokinins. Glycosyltransferase proteins that can glycosylate cytokinins have been described previously. In 2004, Hou et al. found that Arabidopsis UGT76C1 and UGT76C2 can glycosylate cytokinins (Hou et al. 2014); however, since then no new proteins have been reported. In this research, we identified a new protein, Os6, that can glycosylate cytokinins. The $\mathrm{K}_{\mathrm{m}}$ value of Os6 is similar to that of UGT76C1. Therefore, we propose that Os6 in rice has the same function as UGT76C1 in Arabidopsis. In the future we will try to generate transgenic rice overexpressing Os6 to study its function in rice.

The glycosyltransferase Os6 is the first protein to be identified from rice that can glycosylate cytokinins. With the completion of the rice genome, the functions of many rice genes have been determined. However, most of the genes in the rice GT1 family have not yet been identified. There have been limited studies on cytokinin synthesis in rice and, in particular, the downstream pathway of cytokinin glycosylation. Moreover, there have been no new discoveries regarding cytokinin glycosyltransferases in rice. In this project, we identified eight candidate cytokinin glycosyltransferases genes and used LC-MS to determine whether they were functionally active in glycosylating cytokinins. Our results will allow future researchers to investigate the role of the Os6 cytokinin glycosyltransferase in rice.

Os6 was overexpressed in Arabidopsis, and transgenic lines were obtained through three generations of screening. Measurements of the cytokinin glycosides produced by these transgenic lines showed that Os6 is functionally active as a glycosylase in plants. Thus, Os6 is part of the cytokinin metabolic pathway in plants. However, because this study was carried out in the model plant Arabidopsis and involves heterologous expression, our results may not be transferable to rice. Therefore, in future, we plan to examine the function of Os6 in transgenic rice.

A spatiotemporal expression analysis of rice plants showed that Os6 is highly expressed in young organs 


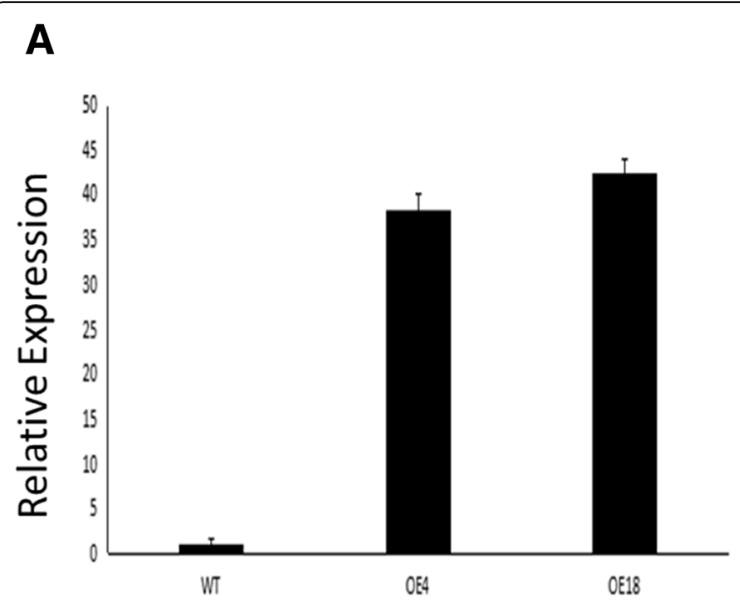

B

Fig. 6 Overexpression Os6 into Arabidopsis and determination their cytokinin glycosides. Overexpression of rice glycosyltransferase gene Os6 into Arabidopsis and determination of cytokinin glycosides in transgenic Arabidopsis. a. The relative expression of Os6 in Arabidopsis. $\mathbf{b}$. The content of cytokinin glycosides in transgenic Arabidopsis

and in leaf sheaths. This suggests that Os6 may play a specific role in these tissues. Transgenic rice overexpressing Os6 will be useful for examining the growth and development of these parts and the distributions of cytokinins.

Cytokinins are one of the five main plant hormones and play very important roles in plants (Larrieu et al. 2015). Here, we examined cytokinin glycosylation and the cytokinin contents in plants. However, cytokinins have important relationships with other plant hormones, including auxin and abscisic acid, that also affect growth and development ( $\mathrm{Li}$ et al.2016). Therefore, research on these hormones may also prove to be important in the future.

\section{Conclusion}

In this study, we cloned the rice glycosyltransferase gene Os6, constructed a prokaryotic expression vector, and expressed and purified the active enzyme protein in bacteria. Using in vitro enzymatic reactions and LC-MS, we found that Os6 can glycosylate cytokinins in vitro. We then overexpressed Os6 in Arabidopsis and extracted cytokinin glycosides from transgenic and WT plants. The transgenic Arabidopsis plants had increased cytokinin glycosides in comparison with the WT-plants, indicating that Os6 can also glycosylate cytokinins in planta and may be involved in the cytokinin pathway of rice.

\section{Methods}

\section{Plant materials and growth conditions}

The rice Nipponbare ecotype was used in this study. Intact and uniformly sized rice grains were surface sterilized with $75 \%$ ethanol for $2 \mathrm{~min}, 0.1 \%$ mercuric chloride solution for $3 \mathrm{~min}$, and then washed 3-4 times with sterile water. The seeds were placed in sterile culture bottles containing a small amount of water for $3 \mathrm{~d}$ until germination.
The seedlings were then transferred to plastic pots and grown in a greenhouse at $30^{\circ} \mathrm{C}$ with natural light $(12 \mathrm{~h}$ light and $12 \mathrm{~h}$ dark).

Arabidopsis ecotype Columbia was used as a plant model in this study. Mature intact wild-type (WT) seeds were sterilized with $0.1 \% \mathrm{Hg}$ for $2 \mathrm{~min}, 75 \%$ alcohol for $3 \mathrm{~min}$, and then rinsed 3-5 times with sterile water. The seeds were placed in Murashige and Skoog medium, vernalized at $4{ }^{\circ} \mathrm{C}$ in the dark for $3 \mathrm{~d}$, and then germinated in a culture chamber at $22^{\circ} \mathrm{C}$ with $16 \mathrm{~h}$ light $(100 \mu \mathrm{mol}$ $\mathrm{m}^{-2} \mathrm{~s}^{-1}$ ) and $8 \mathrm{~h}$ dark for 2 weeks. The seedlings were then transplanted to small pots containing soil and grown under culture chamber conditions as described.

\section{RNA extraction and reverse transcription}

For the RNA extraction, young WT rice plants at the two-leaf and one-heart stage were ground to a powder in liquid nitrogen and $1 \mathrm{~mL}$ of TRIzol reagent was added per $0.2 \mathrm{mg}$ of powder. The sample was then mixed by inversion and, after standing for $5 \mathrm{~min}, 200 \mu \mathrm{L}$ phenol chloroform was added. The sample was vortexed to denature the plant proteins and, after standing for $5 \mathrm{~min}$, was centrifuged at $13,400 \times g$ for $5 \mathrm{~min}$ at $4{ }^{\circ} \mathrm{C}$. The supernatant was transferred to a new centrifuge tube, an equal volume of isopropanol was added, and the sample was mixed by inversion. After standing for $10 \mathrm{~min}$, the sample was again centrifuged for $5 \mathrm{~min}$ at $4{ }^{\circ} \mathrm{C}$, the supernatant discarded, and $1 \mathrm{~mL}$ of $75 \%$ ethanol was added. The flocculated precipitate was washed, and then centrifuged at $13,400 \times g$ for $1 \mathrm{~min}$ at $4{ }^{\circ} \mathrm{C}$. The ethanol was discarded, the sample was centrifuged at $13,400 \times g$ for 1 $\min$ at $4{ }^{\circ} \mathrm{C}$ and the liquid carefully removed using a $20-\mu \mathrm{L}$ pipette. The sample was air dried at room temperature for $5 \mathrm{~min}$ and $20-50 \mu \mathrm{L}$ DEPC water was added to dissolve the nucleic acids. Samples were stored 
at $-20{ }^{\circ} \mathrm{C}$ until required. For the reverse transcription, a reverse transcription kit (TaKaRa, Japan) was used according to the manufacturer's protocol.

\section{Quantitative RT-PCR}

For the first step of the quantitative real-time PCR (qRT-PCR), total RNA was extracted from 2-week-old rice plants using TRIzol reagent. For the second step, reverse transcription from RNA to cDNA, a Primescript RT reagent kit (TransGen, TaKaRa) was used. A $20 \mu \mathrm{L}$ reaction volume was used for the qRT-PCR, which was performed using a Bio-Rad real-time thermal cycling system. SYBR-Green (TaKaRa) was used to detect quantitative gene expression, with ubiquitin used as an internal control. The data were analyzed using Bio-Rad CFX Manager software. The primer information is included in Additional file 2: Table S1.

\section{Plasmid construction and plant transformation}

(1) Candidate gene cloning. RNA was extracted from WT rice and reverse-transcribed into cDNA. Candidate gene sequences were amplified using the primers F:CGCGGATCCATGACAGCACCGATGAC and R:CGGGGTACCCTAGTGTTCTTCCACTC, which were designed using the sequences available on the rice genome website. After amplification, the candidate gene fragment was ligated into an intermediate carrier and its sequence was confirmed by sequencing.

(2) Construction of prokaryotic expression vectors containing candidate genes. To facilitate cloning we modified a common prokaryotic expression vector, PGEX-2 T (Zhang et al. 2008), by inserting a multiplecloning site between the BamHI and EcoRI restriction sites (BamHI-NdeI-NotI-SphI-NcoI-SalI-SacI-XhoIHindIII-EcoRI) and renamed the vector pGEX-3H. Each rice gene was individually cloned into pGEX-3H and the plasmid isolated. After verification by sequencing, the plasmid was transformed into the Escherichia coli expression strain XL1-Blue, to express the fusion protein OS6-glutathione S-transferase.

\section{Purification of cytokinin glycosyltransferase protein and in vitro cytokinin glycosyltransferase enzymatic reactions}

(i) Protein expression was induced in XL1-Blue by incubating the bacteria overnight at $37^{\circ} \mathrm{C}$ and then culturing them to an $\mathrm{OD}_{600}=0.8-1.0$ at $20^{\circ} \mathrm{C}$ with shaking at $180 \mathrm{rpm}$. Isopropyl $\beta$-D-thiogalactoside was added to induce the expression of the protein encoded by the rice gene. (ii) To isolate the target protein, the bacterial cells were collected by centrifugation at $5000 \times g$ for $10 \mathrm{~min}$ at $4{ }^{\circ} \mathrm{C}$. The cells were resuspended in phosphate buffer saline and disrupted by sonification. The solution was applied to a column to isolate the target protein using its glutathione S-transferase tag. A protease was used to elute the protein and obtain a high purity level for the enzyme activity analysis. (iii) For the in vitro enzymatic reactions, $2 \mu \mathrm{g}$ UGT-purified protein was added to a $100-\mu \mathrm{L}$ reaction mixture containing 1 $\mathrm{mM}$ cytokinin, $5 \mathrm{mM}$ UDP-glucose, $50 \mathrm{mM} N^{\prime}-\alpha-$ hydroxythylpiperazine- $N^{\prime}$ - ethanesulfanic acid buffer (pH 7.0) and 14.4 mM 2-mercaptopethanol and incubated at various temperatures for different reaction times.

\section{High-performance liquid chromatography (HPLC)} detection of substrates and $\left(\mathrm{K}_{\mathrm{m}}\right)$ value determinations After the enzymatic reaction, the mixture was filter-sterilized and added to a 5- $\mu \mathrm{m}$ C18 HPLC column $(150 \times 4.6 \mathrm{~mm}$ Zorbax; Agilent, USA) and gradient-eluted at room temperature. Phase A was a filter-sterilized organic solvent in methanol and Phase B was a filter-sterilized $0.1 \%$ phosphoric acid aqueous solution. These were applied to the column as follows: 90\% Phase A and 10\% Phase B for $22 \mathrm{~min}, 35 \%$ Phase A and 65\% Phase B for $25 \mathrm{~min}$, and 90\% Phase A and 10\% Phase B for $35 \mathrm{~min}$. An HPLC spectrophotometer (Shimadzu, Japan) was used with a D2 and set to a 270-nm detection wavelength. For the MS, we used a Shimadzu LC-MS system. The column used was the same as that used for the LC. The Phase A solution was still methanol, while the Phase B solution was $0.1 \%$ formic acid. The heater was set to $180^{\circ} \mathrm{C}$ and the mass spectrometer was operated with a positive electrospray ionization mode of $50 \mathrm{eV}$ and a $5.0-\mathrm{kV}$ probe voltage. Data acquisition and analysis was performed using Xcalibur software (version 2.0.6).

To calculate the $\mathrm{K}_{\mathrm{m}}$ of the glycosyltransferase and its substrate, a Michaelis-Menten kinetic analysis was performed. We used the same reaction system as described above. The reaction was performed in a $30^{\circ} \mathrm{C}$ water bath for $1,5,10$, 15, 20, 30, 40, 60, 90, 120 and $180 \mathrm{~min}$. Reactions were snap-frozen in liquid nitrogen and either used immediately for HPLC or were stored at $20^{\circ} \mathrm{C}$ until required.

To determine the kinetic constants, nine concentration gradients were prepared using substrate concentrations of 0-1 mM, 0.025 mM, 0.05 mM, 0.075 M, 0.1 mM, 0.15 mM, $0.2 \mathrm{mM}, 0.4 \mathrm{mM}, 0.6 \mathrm{mM}, 1.0 \mathrm{mM}$ or $2 \mu \mathrm{g} / \mathrm{mL}$. The proteins being tested were incubated with the substrate and 5 $\mathrm{mM}$ UDP-glucose in the optimum buffer at the optimum $\mathrm{pH}$, temperature and time. The final HPLC results were analyzed and the most suitable $\mathrm{K}_{\mathrm{m}}$ and maximum rate $\left(\mathrm{V}_{\max }\right)$ values were calculated from the kinetic equations.

\section{Generation of transgenic Arabidopsis and cytokinin extraction}

To generate the Os6 overexpression lines, we cloned the full-length Os6 cDNA from rice into the pBI121 binary 
vector, which is driven by the CaMV35S promoter. This was then transformed into to the Agrobacterium tumefaciens GV3103 strain. Strain GV3103 containing the rice glycosyltransferase gene Os6 was activated and grown to a concentration of approximately $\mathrm{OD}_{600}=0.8$. The cells were collected by high-speed centrifugation at $13,400 \times g$ for $10 \mathrm{~min}$ at $4{ }^{\circ} \mathrm{C}$ and diluted in $1 \%$ sucrose to $\mathrm{OD}_{600}=$ 0.1. Then, the Silwet L-77(GE, Beijing) activator was added, and the bacterial solution was used for in plant transformations at room temperature. WT Arabidopsis plants at 4-5 weeks of age were selected for transformation because of their vigorous growth and stage (flowering) in which the stem is $1 \mathrm{~cm}$ in length but the anther has not yet grown. The flower buds were dipped into the Agrobacterium GV3103 solution for $15 \mathrm{~s}$ three times. The plants were then covered with black cloth for $24 \mathrm{~h}$ to allow infection to occur. After flower maturation and seed formation, the seeds were collected, and transformants were selected on medium containing kanamycin. Homozygous transgenic Arabidopsis plants overexpressing Os6 were obtained after three generations of antibiotic selection.

To extract cytokinins from the plant tissues, 2-week-old WT and transgenic Arabidopsis plants were independently ground in liquid nitrogen. Then, $80 \%$ methanol solution was added, and the samples were mixed by inversion at $4{ }^{\circ} \mathrm{C}$ for $24 \mathrm{~h}$. The mixtures were centrifuged at $13,400 \times g$ for $10 \mathrm{~min}$ and the supernatant were collected. The mitogen glycoside solutions were isolated using the described cytokinin HPLC protocol, with UGT76C1 as a positive control.

\section{Additional files}

\begin{abstract}
Additional file 1: Figure S1. Cloning and Construction of the Prokaryotic Expression Vector. The cloning of Rice Glycosyltransferase gene OS6 and the constructing of its prokaryotic expression vetor. A. Cloning Rice Glycosyltransferase gene Os6 from rice gene data bank. B. Constructing of prokaryotic expression vetor of rice glycosyltransferase gene Os6. Figure S2. Phylogenetic tree comparison of UGT76C1 and UGT76C2 with rice cytokinin glycosyltransferase. To further understand the distant relationship of the rice glycosyltransferase phylogenetic tree, we compared the glycosyltransferase UGT76C1 and UGT76C2, which has been shown to be a glycosyl cytokinin in Arabidopsis, to the predicted cytokinin glycosyltransferase phylogenetic tree in rice. (DOCX $9898 \mathrm{~kb}$ )

Additional file 2: Table S1. Primers used in this study. (DOC $31 \mathrm{~kb}$ )
\end{abstract}

\footnotetext{
Abbreviations

APRRs: Arabidopsis pseudoresponse regulators; ARRs: Arabidopsis response regulators; CKX: Cytokinin oxidative dehydrogenase; CRE1: Cytokinin response 1; DMAPP: Dimethyl propylene diphosphate; GST: Goods and Services Tax; GT1: Family 1 glycosyltransferases; HEPES: N'-ahydroxythylpiperazine-N'-ethanesulfanic acid; iPA: Isoamyladenosine; iPDP: Isoamyladenosine-5-diphosphate; iPMP: Adenosine-5-phosphate: IPTG: Isopropyl $\beta$-D-Thiogalactoside; IPTP: Isoprenoid adenosine-5triphosphate; LC-MS: Liquid chromatography-mass spectrometry; MS: Murashige and skoog; PBS: Phosphate buffer saline; qRTPCR: Quantitative real-time PCR; RT: Room temperature; SDS-PAGE: Sodium dodecyl sulfate polyacrylamide gel electrophoresis; tRNA: Transport RNA; WT: Wild-type; ZMP: Trans-zeatin nucleoside phosphate
}

\section{Acknowledgments}

This project was supported by grants from the National Natural Science Foundation of China (Grant No. 21675071 and 31701827), the Natural Science Foundation of Shandong Province Mayor (China, ZR2017CM009), and the Shandong Province Science and Technology Development Plan (J12LE07). We thank Dr. Jennifer Smith, and Dr. Shelley Robison from Liwen Bianji, Edanz Group China (www.liwenbianji.cn/ac), for editing the English text of a draft of this manuscript.

\section{Funding}

This project was supported by grants from the National Natural Science Foundation of China (Grant No. 21675071 and 31701827), the Natural Science Foundation of Shandong Province Mayor (China, ZR2017CM009), and the Shandong Province Science and Technology Development Plan (J12LE07).

\section{Availability of data and materials}

The detail data of the primers used for plasmid construction were list in Additional file 2: Table S1. The rice genome sequences were downloaded from the rice website http://rice.plantbiology.msu.edu.

\section{Authors' contributions}

$\mathrm{PL}, \mathrm{KL}$ and $\mathrm{YJL}$ conceived and designed the experiment. PL, $\mathrm{KL}$ and $\mathrm{RML}$ conducted the experiments, performed data analysis and wrote the manuscript. XRH and SW participated in material development, sample preparation and data analysis. SLJ and BKH drafted proposals and corrected the manuscript. All authors read and approved the final manuscript.

\section{Authors' information}

BK H, Professor in Plant Molecular Genetics, College of Life Science, Shandong University, China.

RM L, Professor and Head of the Instrument Analysis Lab, College of Pharmacy, Liaocheng University, China.

LS J, Professor in Plant Molecular Biology, College of Pharmacy, Liaocheng University, China.

P L, Ph D in Plant Molecular Genetics, College of Pharmacy, Liaocheng University, China; College of Life Science, Shandong University, China. KL, Ph D in Agronomy, College of Pharmacy, Liaocheng University, China. YJ L, Ph D in Plant Molecular Genetics, College of Life Science, Shandong University, China.

XR H, Ph D in Chemistry, College of Pharmacy, Liaocheng University, China. SW, Ph D in Toxicology, College of Pharmacy, Liaocheng University, China.

Ethics approval and consent to participate

Not applicable.

\section{Consent for publication}

Not applicable.

\section{Competing interests}

The authors declare that they have no competing interests.

\section{Publisher's Note}

Springer Nature remains neutral with regard to jurisdictional claims in published maps and institutional affiliations.

Received: 6 December 2018 Accepted: 21 March 2019

Published online: 29 March 2019

\section{References}

Ashihara H, Stasolla C, Fujimura T, Crozier A (2018) Purine salvage in plants. Phytochemistry 147:89-124

Bansal M, Sharma M, Kanwar P, Goyal A (2016) Recent advances in proteomics of cereals. Biotechnol Genet Eng Rev 32(1-2):1-17

Cao PJ, Bartley LE, Jung KH, Ronald PC (2008) Construction of a rice glycosyltransferase phylogenomic database and identification of ricediverged glycosyltransferases. Mol Plant 1(5):858-877

Ciaglia E, Abate M, Laezza C, Pisanti S, Vitale M, Seneca V, Torelli G, Franceschelli S, Catapano G, Gazzerro P, Bifulco M (2017) Antiglioma effects of N6isopentenyladenosine, an endogenous isoprenoid end product, through the downregulation of epidermal growth factor receptor. Int J Cancer 140(4): 959-972 
Frebortova J, Plihal O, Florova V, Kokas F, Kubiasova K, Greplova M, Simura J, Novak O, Frebort I (2017) Light influences cytokinin biosynthesis and sensing in Nostoc(cyanibacteria). J Phycol 53(3):703-714

Hluska T, Dobrew PI, Tarkowska D, Frebortova J, Zalabak D, Kopecny D, Plihal O, Kokas F, Briozzo P, Zatloukal M, Motyka V, Galuszka P (2016) Cytokinin metabolism in maize: novel evidence of cytokinin abundance interconversions and formation of a new trans-zeatin metabolic product with a weak anticytokinin activity. Plant Sci 247:127-137

Hou BK, Lim EK, Higgins GS, Bowles DJ (2004) N-glucosylation of cytokinins by glycosyltransferases of Arabidopsis thaliana. J Biol Chem 279(12):47822-47832

Kaltenegger E, Leng S, Heyl A (2018) The effects of repeated whole genome duplication events on the evolution of cytokinin signaling pathway. BMC Evol Biol 18(1):76

Kurepa J, Li Y, Smalle JA (2014) Cytokinin signaling stabilizes the response activator ARR1. Plant J 78(1):157-168

Lao J, Qikawa A, Bromley JR, Mclnerney P, Suttangkakul A, Smith-Moritz AM, Plahar $\mathrm{H}$ et al (2014) The plant glycosyltransferase clone collection for functionalgenomics. Plant J 79(3):519-527

Larrieu A, Vernoux T (2015) Comparison of plant hormone signalling systems. Essays Biochem 58:165-181

Li G, Ma J, Tan M, Mao J, An N, Sha G, Zhang D, Zhao C, Han M (2016) Transcriptome analysis reveals the effects of sugar metabolism and auxin and cytokinin signaling pathways on root growth and development of grafted apple. BMC Genomics 17:150

Lomin SN, Krivosheev DW, Steklow MY, Arkhipov DV, Osolodkin DI, Schmulling T, Romanov GA (2015) Plant membrane assays with cytokinin receptors underpin the unique role of free cytokinin bases as biologically active ligands. J Exp Bot 66(7):1851-1863

Morris RO, Bilyeu KD, Laskey JG, Cheikh NN (1999) Isolation of a gene encoding a glycosylated cytokinin oxidase from maize. Biochem Bioph Res Co 255(2): 328-333

Morrison EN, Knowles S, Hayward A, Thorn RG, Saville BJ, Emery RJ (2015) Detection of phytohormones in temperate forest fungi predicts consistent abscisic acid production and a common pathway for cytokinin biosynthesis. Mycologia 107(2):245-257

Plihalova L, Vylicilova H, Dolezal K, Zahajska L, Zatloukal M, Strnad M (2016) Synthesis of aromatic cytokinins for plant biotechnology. New Biotechnol 33(5):614-624

Ramegowda V, Basu S, Krishnan A, Pereira A (2014) Rice GROWTH UNDER DROUGHT KINASE is required for drought tolerance and grain yield under normal and drought stress conditions. Plant Physiol 166(3):1634-1645

Smehilova M, Dobruskova J, Novak O, Takac T, Galuszka P (2016) Cytokininspecific glycosyltransferases possess different roles in cytokinin homeostasis maintenance. Front Plant Sci 7:1264

Wang J, Ma XM, Kojima M, Sakakibara H, Hou BK (2011) N-glucosyltransferase UGT76C2 is involved in cytokinin homeostasis and cytokinin response in Arabidopsis thaliana. Plant Cell Physiol 52(12):2200-2213

Xiao GQ, Qin H, Zhou JH, Quan RD, Lu XY, Huang RF, Zhang HW (2016) OsERF2 controls rice root growth and hormone responses through tuning expression of key genes involved in hormone signaling and sucrose metabolism. Plant Mol Biol 90(3):293-302

Xu Y, Burgess P, Huang B (2017) Transcriptional regulation of hormone-synthesis and signaling pathways by overexpressing cytokinin-synthesis contributes to improved drought tolerance in creeping bentgrass. Physiol Plantarum 161(2):235-256

Yamburenko MV, Kieber JJ, Schaller GE (2017) Dynamic patterns of expression for genes regulating cytokinin metabolism and signaling during rice inflorescence development. PLoS One 12(4):e0176060

Yeh SY, Chen HW, Ng CY, Lin CY, Tseng TH, Li WH, Ku MS (2015) Downregulation of cytokinin oxidase 2 expression increases tiller number and improves rice yield. Rice 8(1):36

Yu D, Yu F, Du C, Li X, Zhao X, Liu X (2015) RPN1a, a subunit of the 265 proteasome, controls trichome development in Arabidopsis. Plant Physiol Biochem 88:82-88

Zhang H, Ohyama K, Boudet J, Chen Z, Yang J, Zhang M, Muranaka T, Maurel C, Zhu JK, Gong Z (2008) Dolichol biosynthesis and its effects on the unfolded protein response and abiotic stress resistance in Arabidopsis. Plant Cell 20(7):1879-1898

Zubo YO, Blakley IC, Yamburenko MV, Worthen JM, Street IH, Franco-Zorrilla JM, Zhang W, Hill K, Raines T, Solano R, Kieber JJ, Loraine AE, Schaller GE (2017) Cytokinin induces genome-wide binding of the type-B response regulator ARR10 to regulate growth and development in Arabidopsis. Proc Natl Acad Sci U S A 114(29):E5995-E6004

\section{Submit your manuscript to a SpringerOpen ${ }^{\circ}$ journal and benefit from:}

- Convenient online submission

- Rigorous peer review

- Open access: articles freely available online

High visibility within the field

- Retaining the copyright to your article

Submit your next manuscript at $\boldsymbol{\nabla}$ springeropen.com 